\title{
Recent Progress in Solar or Stellar Interior Modelling
}

\section{S. Turck-Chièze}

Service d'Astrophysique, CEA/DSM/DAPNIA, CE Saclay, Gif sur Yvette, 91191, FRANCE, e-mail:cturck@cea.fr

Abstract. Acoustic modes are well-suited probes to check the internal stellar structure and bring interesting constraints on turbulence regions, mixing in stellar interiors and magnetic fields. The SOHO satellite, through the helioseismic instruments GOLF, MDI and VIRGO, has significantly improved the knowledge of these modes owing to an excellent duty cycle (greater than $90 \%$ ) and the capability to detect very low amplitude modes, down to $3 \mathrm{~mm} / \mathrm{s}$. Five years of the SOHO misson guarantee an accurate view of the solar interior, weakly dependent on the turbulent surface, from the energy-generating core to the surface.

The recent results allow us to verify some of the theoretical assumptions of stellar modelling. The tachocline layers, located in a narrow region at the base of the convection zone, support an hydro (or magnetohydro) dynamical instability which induces mixing and results in ${ }^{7} \mathrm{Li}$ depletion in accordance with photospheric observations. On the contrary, central mixing is not favoured by the present observations. Nuclear reaction rates of the pp chain are now constrained by the behaviour of the sound speed and density. Döppler velocity measurements appear as an excellent technique to follow in real time the temporal evolution of the luminosity produced in the core. Some puzzling questions about dynamical effects in stellar plasmas can now start to be addressed for the first time. The theoretical neutrino emissions can be now directly deduced from our helioseismic vision of the energy-generating core.

Nowadays, helioseismology provides a dynamical vision of the external half of the Sun (20\% in mass), as a result of the extraction of the sound speed, density, rotation profiles and of the time evolution of velocity measurements. Below, the classical static vision of the nuclear region persists because of the poor spatial resolution offered by acoustic modes ( $\pm 6 \%$ in radius, $10 \%$ in mass) and the long integration time (several years). Gravity modes will be extremely useful to improve the spatial resolution in the radiative region. The extended observations with the SOHO satellite may be extremely useful to detect some of these modes.

\section{Introduction}

The theoretical bases of stellar evolution were established in the twenties and thirties by Eddington, Atkinson, Houtermans, Gamow, Weizsäcker, Bethe... Then the theory of stellar evolution was developed vigourously thanks to famous actors such as Chandrasekar, Schwarzschild, Fowler, Henney... The improvement of the computing capabilities has allowed to show the pertinence of these new 
concepts and the impressive progress made by the understanding of the great phases of the life of stars in the Hertzsprung-Russell diagram. Although the undeniable successes of this theory, some unsolved problems clearly show that it is not complete: solar neutrino puzzle, lithium depletion, internal stellar dynamo, evolution of the internal angular momentum, origin of the winds... This is partly due to the fact that two main actors are absent from this formalism: the rotation and the magnetic field which have generally important roles in the dynamical phases of evolution.

Nowadays we begin a new step where the interaction of the stars with the insterstellar medium is particularly studied to understand variability, winds, galactic evolution, and high energy phenomena. This requires a better understanding of the dynamical phases of stellar evolution. The best way to impulse this progress is to develop the seismology of stars which gives a more precise view of the interior of stars. It is extremely fruitful to begin by the best known star, the Sun, due to its proximity and its important role for life on Earth. After intense efforts on the ground, the space-based SOHO mission has demonstrated that we have reached the golden age of helioseismology thanks to the three instruments dedicated to seismology: GOLF (Gabriel et al. 1995, 1997), MDI (Scherrer et al. 1995; Kosovichev et al. 1997), and VIRGO (Frölich et al. 1995, 1997). This star is not dominated by dynamical effects, the effect of rotation and magnetic field are only perturbations of a static structure so its study allows to understand in details the probes used, the fundamental physics and the forgotten processes thanks to the unique detection of several thousands well identified acoustic modes. Logically, the next step will be the development of asteroseismology of solar-like stars with the COROT (first ESA project), MONS and MOST missions and the extensive study of 50000 stars in different environments and mass ranges with EDDINGTON, the second ESA project (Favata, Roxburgh \& Christensen-Dalsgaard 2000). Our objective is to slowly abandon the static vision of stars, well described by a one dimensional model, in favour of a dynamical vision of stars including hydrodynamics and a 3-D representation.

\section{The helioseismic context}

Four quantities are potentially accessible to seismology with acoustic modes: the radial sound speed, density, rotation and magnetic field. The great challenges of helioseismology are the small amplitudes of the modes and the accuracy required in order to check the solar interior. This point is partly illustrated in the reviews of J. Schou and R. Howe (these proceedings) who show the complexity of the analysis of the high degree modes and the impressive requirements for an observation of the dynamics of the rotation below the convection zone. These contributions illustrate the need for sophisticated techniques of data analysis and inversion to extract local information throughout the Sun.

I would like to add here a third difficulty connected to the knowledge of the core which begins to be properly accessible with the SOHO satellite after extremely impressive results obtained with ground networks such as BiSON and IRIS. If it is natural to extract information of the less dense layers of the Sun at radius $>0.6 R_{\odot}$ from acoustic modes, it would also be natural to extract information of the nuclear core from gravity modes as they are 5 to 10 times 
more sensitive to the physics of the core. However, these modes are known to have very low amplitudes in a very noisy range of frequencies. With the extreme quality of the $\mathrm{SOHO}$ satellite, the detection limit has been improved by a factor of at least 10 as a result of the improved duty cycle and stability, of the quality of the instruments and by avoiding perturbations in the observations. If it is not possible to have today a core vision through gravity modes, it allows already a real insight on the nuclear core thanks to more than 100 detected global acoustic modes. Of course this progress is accompagnied by strict requirements on accuracy in order to extract confidently the information on the core. The sound speed varies smoothly from about $500 \mathrm{~km} / \mathrm{s}$ in the central region down to $7 \mathrm{~km} / \mathrm{s}$ at the surface and evolves only slowly during the hydrogen burning phase (Turck-Chièze 2000). This has evident consequences:

- the travel time of a radial mode in the core is only $5 \%$ of the travel time inside the whole Sun.

- the sensitivity of the sound speed to any variation of a fundamental quantity is small. A variation of several \% on a physical parameter has an effect on the sound speed of several $0.1 \%$ roughly (Turck-Chièze et al. 2000a [TC2000a]). An immediate consequence is that if we would like to understand some of the differences between the Sun and the models we need to get an accuracy on the sound speed in the solar core a factor of 10 better, i. e. at the level of $10^{-4}$.

Consequently the frequency of any mode penetrating in the solar core must be known with a relative accuracy of about $10^{-5}$ for it to give useful information on the core. This is formally possible with the techniques which are used (spectral analysis of the residual velocity signal) as the accuracy depends on the integration time. However, any source of bias which may destroy this potential accuracy must be studied. Two types of biases have been studied aboard SOHO: the dependence of the frequency shape on the observation technique adopted (Toutain et al. 1997; Thiery et al. 2000), and the role of the surface turbulent motions and its time evolution on the frequency of the modes (García et al. 2000; TC2000a).

A mode is described by three numbers: the degree $\ell$ corresponds to the number of reflexions at the surface, the order $n$ to the number of nodes along the solar radius and the azimuthal number $m$ informs on the asphericity and the magnetic field. For a given low degree mode, there is potentially more than 40 accessible orders. The higher order modes correspond to the most penetrating acoustic waves but they are also more sensitive to the surface and to its perturbations. The lifetime of these modes is small, their linewidth consequently large and the identification not always easy. As a result the global acoustic modes of high orders are generally determined at several $10^{-4}$ (Lazrek et al. 1997). On the other hand, the lowest order modes are now known at about $10^{-5}$ $(n<10, \nu<1.5 \mathrm{mHz})$, they have longer lifetimes in spite of having a very low amplitude down to $3 \pm 2 \mathrm{~mm} / \mathrm{s}$ (Bertello et al. 2000a,b; García et al. 2000).

The progress made with SOHO gives us confidence that we are able to describe the solar interior through acoustic modes. In the following sections, I shall show the progress done in fundamental physics (section 3), on the dynamical vision of the Sun (section 4), and on the extraction of interesting properties of the nuclear solar plasma (section 5). 


\section{Fundamental physics}

In parallel to the measurements of acoustic modes, large refined progress has been achieved in the nuclear and atomic physics included in the equations of stellar structure. Three types of improvements are interesting to mention:

- two types of laboratory experiments have been performed. Opacity checks of the photon matter interaction at low densities with laser experiments are beginning to be useful. The measurement of the opacity coefficients in the conditions corresponding to the outer layers of the Sun, for some specific elements such as iron, has confirmed an error of a factor 2 on past opacity calculation, due to the difficulty to take properly into account a large number of lines (Springer et al. 1992). Nuclear experiments have been performed near low energy accelerators. The $\left({ }^{3} \mathrm{He},{ }^{3} \mathrm{He}\right)$ cross section has been measured for the first time at an energy typical of the solar plasma (Junker et al. 1998) but no resonance has been discovered at low energy. $\left({ }^{7} \mathrm{Be}, \mathrm{p}\right)$ has also been verified in several ways (Motobayashi et al. 1994; Hammache et al. 1998). This last measurement has confirmed the reduction of $20 \%$ recommended by Turck-Chièze et al. (1993).

- theoretical calculations have improved the extrapolation of the nuclear reaction rates at solar energy. A detailed compilation of all the nuclear reaction rates useful for studies of the Sun has been performed by Adelberger et al (1998). This compilation shows a general accuracy of about $10 \%$ on pp chain reactions and slightly worse on CNO cycle. Several studies have also been dedicated to the problem of screening on not totally weak nor strong coupling plasmas (Dzitko et al. 1995; Gruzinov 1998; Shaviv and Shaviv 1999, 2000; Tsytovich and Bornatici, 2000). A large and continuous effort has been devoted to the detailed calculations of opacity coefficients in order to get coherence between equation of state and opacity as well as expanding the number of elements included in the calculations. Several groups have worked in parallel on this extremely complex problem (Iglesias and Rogers (1996); Seaton (1997); Tsytovich et al. (1996, 2000 ) and many comparisons have been performed. Finally the group at Livermore has delivered to the stellar community tables generated for different conditions of temperature, density and composition (Iglesias and Rogers 1996) and the corresponding equation of state (Rogers, Swenson \& Iglesias 1996). The opacity calculations now include 21 elements from hydrogen to nickel to reach a quality of several \% in relative values in order to offer to the helioseismic community a high level of description of the solar plasma (Rogers and Iglesias 1998). At temperatures below $10000 \mathrm{~K}$, molecules and neutral ions must also be included in the treatment of the radiation transfer. Several methods have been developed and are used to solve the structural equations in this domain (Kurucz 1991; Alexander \& Ferguson 1994).

- the first convincing results of helioseismology, obtained before the SOHO launch, were the determination of the photospheric helium and the precise determination of the base of the convection zone. These results demonstrated that a fundamental process needed to be introduced in the equations of stellar evolution: the slow settling of the elements during the evolution of stars. Detailed calculations have been performed to describe such a process, including gravitational settling and radiation effects, element per element depending on their degree of ionization (Michaud \& Proffitt 1994; Thoul, Bahcall \& Loeb 1994; Turcotte et al. 1998). Such a process (hereafter called microscopic diffusion) is 
now directly introduced in all the solar models and is responsible for more than half of the discrepancy between the Sun and the model noticed in the nineties, see figure 1 Left (Brun, Turck-Chièze and Morel 1998).

As the Sun represents the first opportunity of a direct verification of the stellar evolution equations, a large number of comparisons have been done between the different groups involved in the progress of one or another facet of the calculation (Turck-Chièze et al. 1998). Efforts of this kind guarantee the stability of the results and the limited influence of the numerical uncertainties.
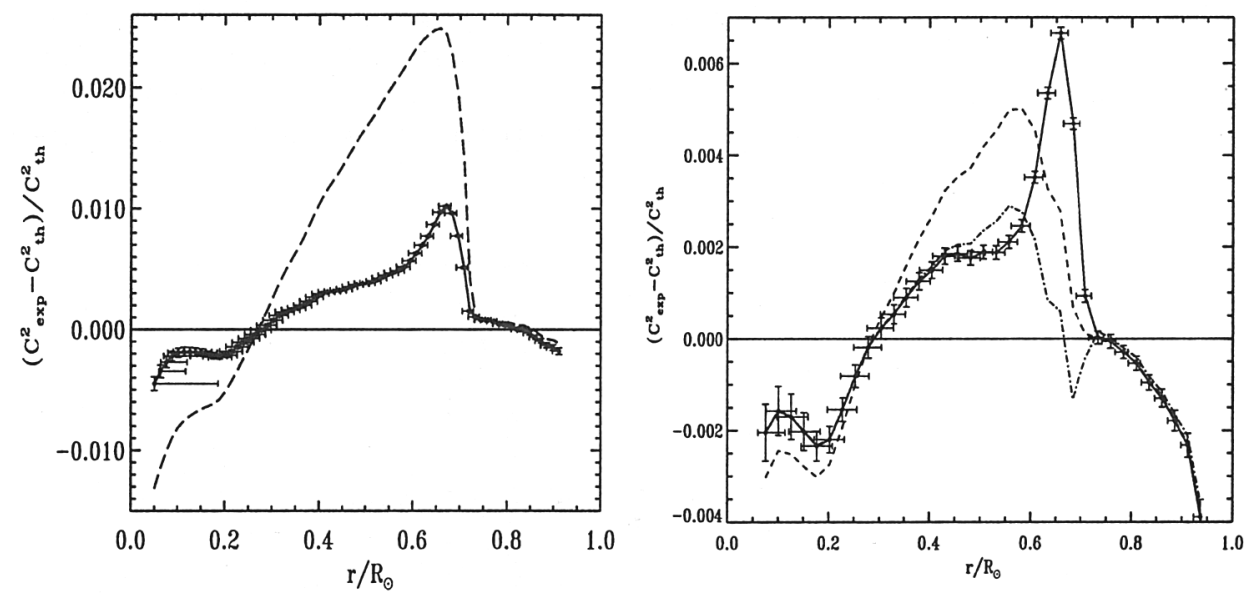

Figure 1. Left: Sound speed square difference between the Sun as measured by seismic experiments (1 year of data integration) and models without (dashed line) and with microscopic diffusion (solid line with error bars) (from Brun, Turck-Chièze and Morel (1998). Right: Sound speed square difference between the Sun observed by GOLF + MDI ( 2 years) and a model including microscopic diffusion (solid line) and two other models including in addition a turbulent term: the dashed line model is calibrated in $\mathrm{Z} / \mathrm{X}$ according to the mean value photospheric observations and the dash-dotted line model is not but the result stays compatible with the observed uncertainty: $\mathrm{Z}$ initial $=\mathrm{Z}$ reference $=0.01959$ (from Brun, Turck-Chièze and Zahn 1999).

\section{A dynamical vision of the Sun down to $0.6 \mathrm{R} \odot$}

Acoustic modes are perfectly adjusted to study this solar region which represent less than $20 \%$ of the solar mass. We must separate it in two parts.

The most external, the outer $0.5 \%$ of the radius is not presently well described by the classical stellar evolution codes. In fact, only a 3-dimensions code can describe the turbulence of the external layers, coupled to a high variation of the rotation and to the presence of magnetic field. Complex simulations of the solar convection in rotating spherical shells have this objective in the future. Nowadays, this leads to difficulties in getting correct absolute values of the frequencies of the acoustic modes which reflect very high at the surface because this part of the Sun is not properly described in the classical framework of stellar evolution. This area is excluded in all the inversions done up to now, as it is visible on the different figures. 
The spatial resolution of the inferred sound speed is smaller than $1 \%$ in radius and the accuracy is of the order of several $10^{-5}$ in the region between 0.6 and $0.99 R_{\odot}$. The region above $r>0.71 R_{\odot}$ is the seat of convection and is now starting to be studied in details through $3 \mathrm{D}$ calculations. Nevertheless the convection in this part of the Sun is totally adiabatic and a simple representation as generally introduced in classical stellar evolution is sufficient to describe the main thermodynamical quantities. At the base of the convection zone, one observes a rapid supression of the differential rotation in a small region spanning less than $0.05 R_{\odot}$ which is called the tachocline (Kosovichev et al. 1997). One clearly sees on figure $1 \mathrm{~b}$ that this region is not properly described by classical models. It is the first time that we observe in a star such a transition between radiation and convection, and puts in evidence, quantitatively, shear layers dominated by horizontal motions. This region is probably the seat of a (magneto) hydrodynamical instability.
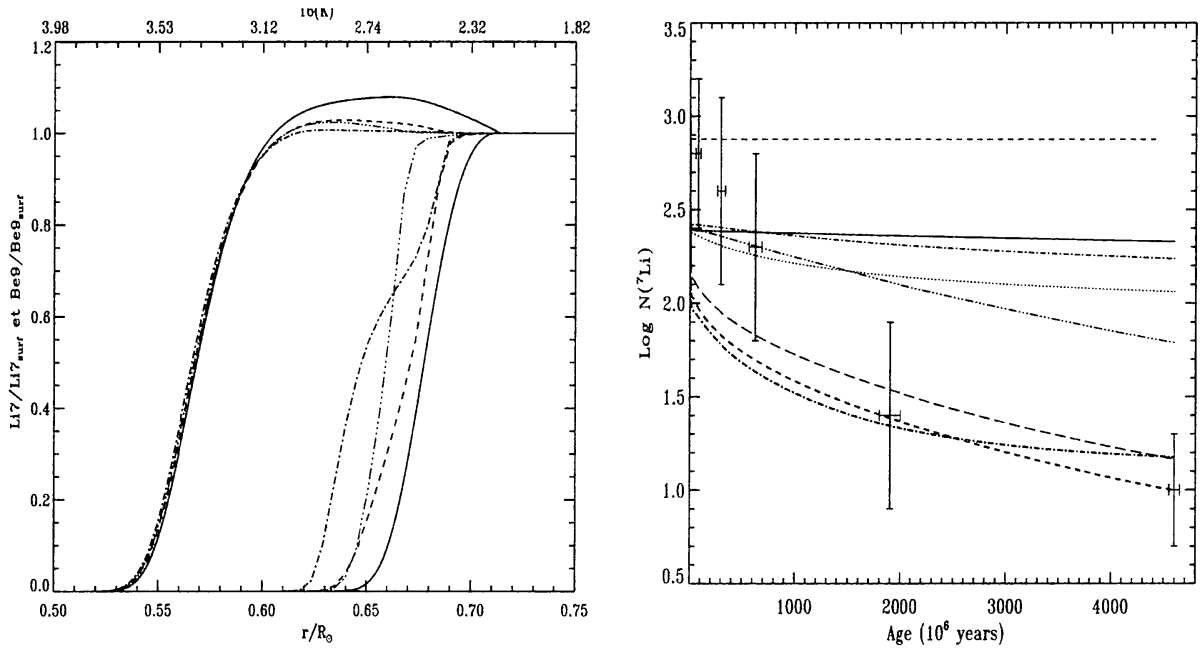

Figure 2. Left: ${ }^{7} \mathrm{Li}$ (right curves) and ${ }^{9} \mathrm{Be}$ (left curves) radial profile for several models as a function of the temperature in $10^{6} \mathrm{~K}$ and the radius. The solid lines correspond to a model without turbulence. All the other models, including turbulence, show a variation of the burning for ${ }^{7} \mathrm{Li}$ but not for ${ }^{9} \mathrm{Be}$. Right: Time-dependent depletion of the ${ }^{7} \mathrm{Li}$ for different models: a model without microscopic diffusion (dashed line), a model including this process (solid line). The dash-dotted line, dash-triple-dotted line and small points line models include turbulence at the base of the convective zone with the prescription corresponding to the present Sun. The other models (dotted line, long-dashed line and thick-dash-dotted) include also a time dependence of the tachocline corresponding to the evolution of the rotation. Superimposed are different open cluster observations: Pleiades, UMaG, Hyades, NGC752 and the Sun. (From Brun, Turck-Chièze and Zahn 1999).

We have proposed a way to model it in adding a turbulent term in the diffusion equation describing only the hydrodynamical character of this insta- 
bility, following the formalism of Spiegel and Zahn (1992). The latter depends on the external rotation and the width of the shear. Such an instability partly inhibits the microscopic diffusion by about $10 \%$ and extends the region of mixing inside the radiative zone (fig $1 \mathrm{~b}$ ). A test to verify such an effect is to follow the abundance of ${ }^{9} \mathrm{Be}$ and ${ }^{7} \mathrm{Li}$ as these elements are burned respectively at a temperature slightly above or corresponding to the temperature of this region (fig 2a). Comparing the destruction of lithium in different stars, we demonstrate that one needs to correlate this zone of instability to the evolution of the rotation along the main sequence to properly reproduce the order of magnitude of lithium destruction (fig $2 \mathrm{~b}$ ) and to avoid any destruction of beryllium (fig 2a). From Brun, Turck-Chièze and Zahn 1999.

It is worth mentioning that after the introduction of microscopic diffusion and the turbulent term at the base of the convective zone, the photospheric abundances of all elements, including helium, are reproduced in the model.

No determination of the magnetic field at the base of the convective zone is obtained up to now. So we cannot yet conclude on the detailed description of this instability and on the proper description of the scenario of the solar cycle. Theoretical investigations are dedicated to this crucial study (Gough 1999; Gilman 2000).

\section{The static vision of the solar core}

The region between $0.3 R_{\odot}$ and $0.6 R_{\odot}$ is mainly dominated by the role of the chemical composition and consequently by the opacity coefficients. This region is also indirectly sensitive to what happens in the core as the different layers react to the production of energy or to any central perturbation. As was mentioned in the second section, the energy-generating core can be tested reasonably accurately for the first time through the sound speed profile as a result to the extended number of global acoustic modes detected with GOLF and MDI (Bertello et al. 2000a,b; García et al. 2000) as well as the refined understanding of the different biases (Basu et al. 1999; Turck-Chièze et al. $2000 \mathrm{~b}$ ). As a result, even though the spatial resolution is insufficiently good $\left( \pm 0.06 R_{\odot}\right.$ or about $\left.\pm 0.1 M_{\odot}\right)$ to properly search for instabilities or to describe correctly the very internal rotation, we begin to answer to some open questions which have been adressed in the last 10 years. In fact, many physical processes can be tested in the light of the present results. In TC2000a, we have examined two kinds of ideas:

- the presence of turbulent mixing in the very central region. We conclude that a relevant central mixing in order to modify the composition of ${ }^{7} \mathrm{Be}$, adjusted to explain the Superkamiokande neutrino results, is not at all supported by the present analysis.

- the sensitivity of the sound speed to the nuclear reaction rates. In this case, we wanted to check if the present results might support a large variation of the assumed screening coefficients, introduced in the determination of the nuclear rate to describe the acceleration process due to the presence of free electrons and neighbour ions. This is a way to test the dynamical effects suggested by Shaviv and Shaviv $(1999,2000)$ or Tystovitch and Bornatici (2000). We conclude that the present results show a great sensitivity to the reaction rates of the pp chain 
(except the interaction of ${ }^{7} \mathrm{Be}$ with proton) which produces $98.5 \%$ of the total luminosity. On the contrary the present sound speed profile is not really sensitive to the CNO cycle.
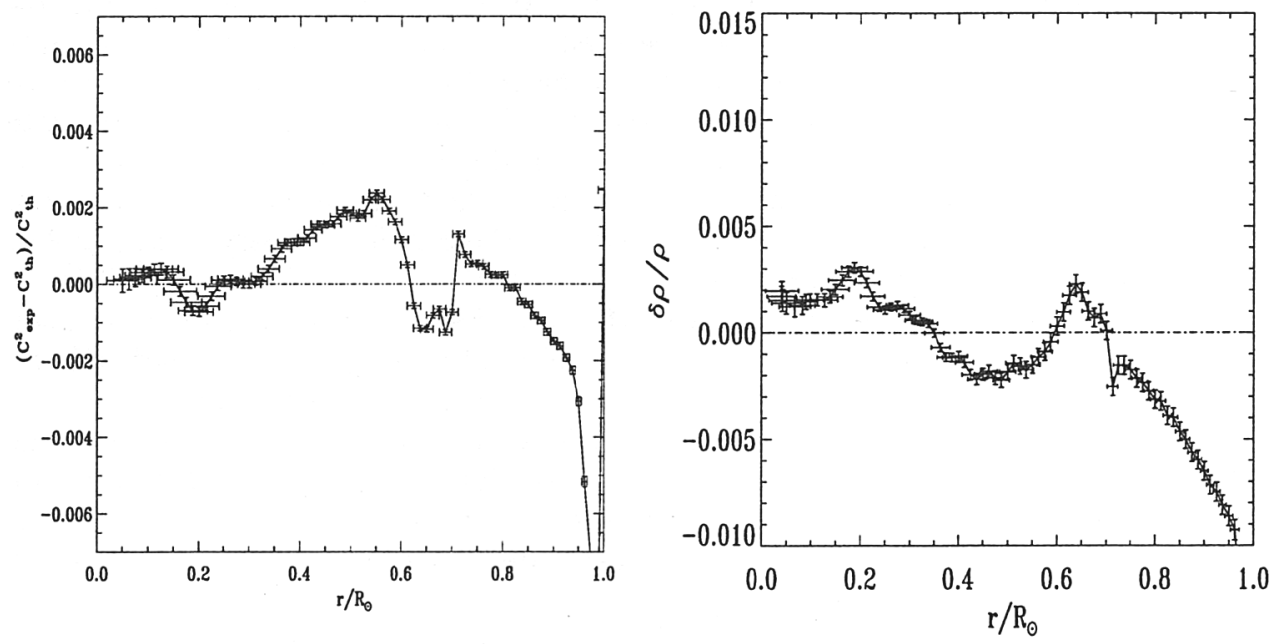

Figure 3. Left: Sound speed square difference between the Sun observed by GOLF + MDI (4 years) and a model including updated fundamental physics, microscopic diffusion, turbulence at the base of the convective zone and an increase of $(p, p)$ by $2 \%$ (from Turck-Chièze et al. 2000a). Right: idem for the density profile (from Turck-Chièze et al. 2000b).

This observation has two interesting consequences:

- We can derive from the persistent small difference between the solar sound speed profile and the model's prediction a variation limit of the pp reaction rate $(+2 \%)$ and a variation limit of the $\mathrm{CNO}$ composition $(+3 \%)$. From that we deduce a seismic model which shows a practically null variation between the Sun and the model for the sound speed and the density profile illustrated by figure 3.

- We can deduce from this seismic model the flux of emitted neutrinos which remains slightly greater than the number of neutrinos detected on Earth. For gallium: $125.5 \mathrm{SNU}$ (121 SNU) must be compared to $76 \pm 8 \mathrm{SNU}$ for the GALLEX and SAGE detections; for chlore: 6.72 SNU (6.07 SNU) must be compared to the Homestake detection of $2.55 \pm 0.25$ SNU, for water: 4.72 (3.81) $10^{6}$ neutrinos $\mathrm{cm}^{-2} \mathrm{~s}^{-1}$ must be compared to the Superkamiokande measurement of $2.44 \pm 0.32$ neutrinos $10^{6} \mathrm{~cm}^{-2} \mathrm{~s}^{-1}$. The values in parentheses correspond to a reduction of $65 \%$ of the $\mathrm{CNO}$ reaction rates and stay compatible with the seismic results. $1 \mathrm{SNU}$ is the solar neutrino unit which corresponds to $10^{-37}$ capture $\mathrm{cm}^{-2} \mathrm{~s}^{-1}$. These conclusions allow the introduction of some unknown properties of the electronic neutrino, e. g. a non zero mass and perhaps a small magnetic moment. The idea that there is some interaction between the neutrino and the Sun is supported by the observation of a variability of the detected neutrino flux with time which could be connected to the solar rotation or to some changes during the solar cycle (Sturrock et al. 1999, 2000). 
We note on figure 3 that the internal part of the Sun seems now better understood than the external one in which we need to inject turbulence and magnetic field.

\section{Perspectives}

The prospect of describing the nuclear core in more details stays a very interesting objective of the extended SOHO mission. We hope to advance our knowledge of internal rotation of the very inner core which could in return inform us on a residual trace of the initial rotation and contribute to the understanding of the internal evolution of the angular momentum. As we have reached the frontier between gravity modes and acoustic modes in detecting a radial mode of order 3 and maybe 1 , we could have the opportunity to detect the first gravity modes with the SOHO satellite and perhaps also learn something about the very internal magnetic field. An upper limit of $1 \mathrm{~cm} / \mathrm{s}$ for the amplitude of gravity modes has been obtained by the analysis of 2 continuous years of MDI, VIRGO and longer series from BiSON (Appourchaux et al. 2000). A new search strategy has led the GOLF team to propose some candidates at a level of no more than 6 $\mathrm{mm} / \mathrm{s}$ (Turck-Chièze et al. 2000c) after 4 years of integrated time. A longer series could be determinant to conclude. Nevertheless the interest of these modes is clearly to gain in spatial resolution and to be able to follow them with time to definitively answer the question: is the solar core dynamic or static as we believe in the classical representation of stars? It appears clear that a second generation of instruments, after the SOHO mission, would be useful to answer to this question because the forest of the gravity modes which is particularly determinant, is probably a factor 10 below the present detection limit of GOLF and MDI (Kumar et al. 1996).

Various efforts are already tackling this problem of detectability in order to gain a definitive insight view of a star from the core to the surface (see for example Turck-Chièze et al. 2000d). If we succeed in improving the signal/noise ratio by a factor 10 (detection of modes of the order of $0.1 \mathrm{~mm} / \mathrm{s} !$ ), it could become a very interesting way to follow in real-time the temporal evolution of the solar energy generation. This would be a fundamental way to study the role of the Sun in the Sun-Earth relationship and in the problem of the evolution of the Earth's climate, even much better than to follow the photospheric luminosity which tells us of the emission of the photons from the core only one million years after the fact. But much progress is needed to reach such an extremely exciting goal.

Acknowledgments. I would like to express my gratitude to the three PIs of the helioseismic instruments aboard SOHO: A. Gabriel for GOLF, P. Scherrer for MDI and C. Fröhlich for VIRGO and to all my colleagues scientists and engineers of the GOLF team who have contributed to obtain so beautiful measurements. The GOLF experiment is based upon a consortium of institutes: the IAS (France), the CEA (France), the IAC(Spain), and the observatories of Bordeaux and Nice (France). I am also particularly grateful to my direct collaborators who have contributed to the improvement of the theoretical description of this simple star: A. S. Basu, G. Berthomieu, S. Brun, J. Christensen-Dalsgaard, 
S. Couvidat, S. Kosovichev, P. Nghiem, J. Provost, F. Rogers, S. Turcotte, J.P. Zahn.

\section{References}

Adelberger, E. et al. 1998, Rev. Mod. Phys, 70, 1265

Alexander, D. R. \& Ferguson, J. W. 1994, ApJ, 437, 849

Appourchaux, T., Frölich, C., Andersen, B. et al. 2000, ApJ, 538, 401

Basu, S., Turck-Chièze, S., Berthomieu, G. et al. 2000, ApJ, 535, 1078

Bertello L., Henney C.J., Ulrich R.K., et al. 2000a , Ap. J., 535, 1066

Bertello L., Varadi F, Ulrich R.K., et al. 2000b, ApJ. lett., 537, L143

Gabriel, A. H., Grec, G., Charra, et al. 1995, Sol. Phys., 162, 61

Brun, A. S., Turck-Chièze, S., \& Morel, P. 1998, ApJ, 506, 913

Brun, A. S., Turck-Chize, S., \& Zahn, J. P. 1999, ApJ., 525, 1032

Dzitko, H., Turck-Chièze, S., Delbourgo, P., \& Lagrange, G. 1995, ApJ, 447, 428

Favata, F., Roxburgh, I., Christensen-Dalsgaard, J. 2000, ESA-SCI 8

Frölich, C. Romero, J., Roth, C., et al. 1995, Sol. Phys., 162, 101.

Frölich, C., Andersen, B. N., Appourchaux, T. et al. 1997, Sol. Phys., 170, 1

Gabriel, A. H., Charra, J., Grec, et al. 1997, Sol. Phys., 175, 207

García, R. A., Régulo, C., Turck-Chièze, S., et al. 2000, Sol. Phys. submitted

Gilman, P. A. 2000, ApJ, 544, L79

Gough 1999, in Helioseismic diagnostics of solar convection and activity, Stanford

Gruzinov A. V. 1998,ApJ, 469, 503

Hammache, F. et al. 1998, Phys. Rev. Lett. 80, 928

Iglesias, C. A. \& Rogers, F. J., 1996, ApJ, 464, 943

Junker, M., et al. 1998, Phys. Rev. C 57, 2700

Kosovichev, A. G., Schou, J. Scherrer, P. H. 1997, Sol. Phys. 1997, 170, 43

Kumar, P., Quataert, E. J., \& Bahcall, J. N. 1996, ApJ, 458, L83

Kurucz, R. L.,Stellar Atmospheres, Kluwer, Dordrecht, 1991, 441.

Michaud, G., \& Proffit, C.R., Inside the stars IAU 137, p 246

Motobayashi, T. et al. 1994, Phys. Rev. Lett., 73, 2680

Rogers, F. J. and Iglesias, C. A. 1998, Space Sc. rev. 85, 61

Rogers, F. J., Swenson, J. \& Iglesias, C. 1996, ApJ, 456, 902

Scherrer, P. H., Bogart, R. S., Bush, R. I., et al. 1995, Sol. Phys., 162, 129

Seaton, M. J. 1997, MNRAS, 289, 700

Shaviv, G. \& Shaviv, N., 1999, Phys. Rep., 311, 99; 2000, Astroph. J., 529, 1054

Spiegel, E. A. \& Zahn, J. P. 1992, A\&A, 265, 106

Springer, P.T. et al. 1992 Phys. Rev. Lett., 69, 3735

Sturrock, et al. 1998, ApJ, 507, 978; ApJ lett., 523, L177; 2000, submitted

Thiery S., Boumier, P., Gabriel A.H., et al. 2000, Astron. Astrophys., 355, 743

Thoul, A., Bahcall, J. N., Loeb, A 1994, ApJ, 421, 828

Toutain, T., Appourchaux, T., Baudin, et al. 1997 Solar Phys., 175, 311

Tsytovich, V. N. \& Bornatici, M. 2000, Plasma Phys. Rep., 89

Tsytovich, V., Bingham, R., De Angelis, U., \& Forlani, A. 1996, Phys. Script. 54, 313

Turck-Chièze, S. 2000, Nucl. Phys. B (Proc. Suppl.), 80, 183

Turck-Chièze, S., et al. 1993 Physics Rep., 230 (2-4), 57-235 
Turck-Chièze, S., Basu, S., Berthomieu, et al. 1998, ESA SP-418, 555

Turck-Chièze, S., Nghiem, P., Couvidat, S., Turcotte, S. 2000a, Sol. Phys., accepted

Turck-Chièze, S., Kosovichev, A. G., Couvidat, S., et al. 2000b, ESA SP-464, in press

Turck-Chièze, S., Garcia, R. A., Couvidat, S. et al. 2000c, ApJ, submitted

Turck-Chièze, S., Robillot, JM., Dzitko, H. et al., 2000d, ESA SP-464, in press

Turcotte, S. et al. 1998, ApJ, 504, 539 